\section{Hier steht eine Anzeige.}

Springer

Photophobie bei Migräneattacken

\title{
Farbspezifische Ausprägung
}

Fragestellung: Gibt es bestimmte Farben, welche die Symptome einer Migräneattacke verstärken oder abschwächen?

Hintergrund: Nahezu alle Patienten mit Migräne weisen während einer akuten Migräneattacke eine erhöhte Lichtempfindlichkeit auf. Diese wird üblicherweise einer verstärkten Empfindlichkeit des visuellen Kortex zugeschrieben. Die mögliche Rolle der Retina und der Umschaltung der visuellen Signale im Thalamus wurde bisher noch nicht gut untersucht.

Patienten und Methodik: Die Arbeitsgruppe aus Boston untersuchte insgesamt 69 Migränepatienten. Sie wurden während akuter Migräneattacken verschiedenfarbigem Licht ausgesetzt und anschließend wurde gemessen, ob die entsprechende Farbe die Kopfschmerzsymptome unbeeinflusst lässt, verstärkt oder abschwächt. Zusätzlich wurde bei den Patienten eine Elektroretinografie mit unterschiedlichen Farben durchgeführt und es wurden visuell evozierte Potenziale abgeleitet.

Ergebnisse: Die psychophysischen Experimente zeigten, dass weißes, blaues, oranges und rotes Licht intensitätsabhängig Kopfschmerzen verstärkten. Dieselben Stimuli konnten auch eine vermehrte Muskelanspannung und pochende Kopfschmerzen auslösen. Bei einem Teil der Betroffenen führte grünes Licht nur zu einer geringen Verstärkung der Kopfschmerzintensität und bei einigen Patienten sogar zu einer Abschwächung der Kopfschmerzen. Diese Ergebnisse konnten durch elektroretinografische Untersuchungen und die Ableitung der visuell evozierten Potenziale repliziert werden. Wurden die Patienten grünem Licht ausgesetzt, waren die evozierten Potenziale am kleinsten.

Noseda R, Bernstein CA, Nir RR et al. Migraine photophobia originating in cone-driven retinal pathways. Brain 2016; 139: $1971-86$
Schlussfolgerungen: Patienten, die während einer Migräneattacke grünem Licht ausgesetzt werden, erfahren eine Abschwächung ihrer Kopfschmerzen, bei allen anderen Farben kommt es zu einer Zunahme der Kopfschmerzen.

\section{- Kommentar von Hans-Christoph Diener, Essen}

\section{Interessanter und wichtiger Ansatzpunkt}

Diese Studie der Arbeitsgruppe aus Boston ist außerordentlich wichtig für das Verständnis der Entstehung der Lichtempfindlichkeit bei der Migräne. Nimmt man die psychophysischen und elektrophysiologischen Untersuchungen zusammen, sieht es so aus, als wenn die Retina und die Verschaltung der Farbsignale im Thalamus bei der Entstehung der Photophobie während Migräneattacken eine wichtigere Rolle spielen als der visuelle Kortex.

Früheren Erfahrungen beziehungsweise Untersuchungen zufolge können lichtempfindliche Migränepatienten eine deutliche Linderung ihrer Kopfschmerzen durch das Tragen einer Sonnenbrille erreichen. Klinisch ist allerdings bisher nicht erprobt worden, ob die Exposition gegenüber grünem Licht tatsächlich die Kopfschmerzintensität reduzieren kann. Das wäre ein interessanter und wichtiger Ansatzpunkt für eine nicht medikamentöse Behandlung. 